\title{
The ethics of global clinical trials
}

\author{
In developing countries, participation in clinical trials is sometimes the only way to access medical \\ treatment. What should be done to avoid exploitation of disadvantaged populations?
}

\author{
Katrin Weigmann
}

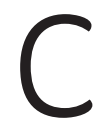
linical research by academic institutions and pharmaceutical companies has followed the general trend of globalization and has moved inexorably towards low- and middle-income countries (LMIC). This trend has raised various concerns, including whether the research being conducted is of value to public health in these countries or whether economically disadvantaged populations are being exploited for the benefit of patients in rich countries. Nevertheless, clinical trials and the research and health care that accompany them can directly benefit patients, in particular those who would otherwise have no or only little access to health care services. It is therefore a matter of striking a fine-tuned balance between the economic and research interests of pharmaceutical companies and academia and the needs of patients in LMIC to make sure that all sides benefit.

cc

... clinical research also helps to build research and health care capacity and can improve local infrastructure and boost the economy"

Clinical trials are a necessary step in drug development and are conducted throughout the world, both in developed and in developing countries. Trials themselves are thus not per se immoral, and there are a variety of reasons to conduct responsible clinical trials in LMIC. Doing so, for example, is often the only way to test drugs and vaccines for diseases that predominantly afflict people in these countries; trying to test the safety and efficacy of a malaria vaccine in Europe or North America would be relatively futile given the lack of patients. Beyond the obvious and direct public health benefits - in terms of both new knowledge and new treatments-clinical research also helps to build research and health care capacity and can improve local infrastructure and boost the economy. In fact, many developing countries have been actively trying to attract clinical research for these reasons.

From the perspective of those conducting the trials, a major benefit of LMIC is that it is easy to enrol patients who are willing to participate, particularly if they are poor. For many of these patients, clinical trials are their only access to medical care. However, it is precisely this unmet medical need that poses a risk for exploitation. "You want to make sure that you don't take advantage of deprivation and that you don't see the world as a convenient population of sick people where you can go and get the data that you need to make more robust health systems in high income countries", commented Alex John London, Professor of Philosophy and Director of The Center for Ethics and Policy at Carnegie Mellon University in the USA. Indeed, human subjects research in LMIC has often been criticized for exporting the risk of research to those who will, in the end, not be able to afford the resulting medical products. Clinical trials can pose real health risks to participants, as the safety and side effects of the drugs or vaccines being tested are not yet fully known.

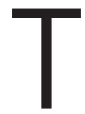

he history of clinical trials and their subsequent globalization can be traced back to the thalidomide scandal in Germany in the early 1960s. Many children were born with severe deformations of their extremities; it subsequently became clear that thalidomide, a sedative developed by the German company Grünenthal, caused birth defects in babies whose mothers had been taking the drug during pregnancy. Public outrage over the devastating effects of the drug and the fact that it had not been sufficiently tested for safety fuelled discussion within the US Food and Drug Administration (FDA) and quickly led to legislation to improve the safety testing of new drugs. The so-called KefauverHarris Amendments were passed to prevent another thalidomide disaster and-although not directly related to the drug scandalintroduced the requirement for drug manufacturers to prove drug effectiveness in addition to safety.

"From the perspective of those conducting the trials, a major benefit of LMIC is that it is easy to enroll patients who are willing to participate, particularly if they are poor"

As the standards for safety and efficacy developed over the decades, so did research ethics principles. Again, it was medical scandals that spawned today's human subjects regulations in the USA, most notably the socalled Tuskegee Syphilis study. In this infamous experiment, conducted from 1932 to 1972, US physicians denied penicillin, a known treatment for syphilis since the late 1940s, to a cohort of syphilis-infected African American sharecroppers to study the natural course of advanced syphilis. Outrage over these practices led to the passage of the 
National Research Act in 1974 and eventually to the Belmont report, which forms the basis for any human subject research conducted in the USA. As a result, the regulatory process for drug development and testing in the USA has very high standards, but at a price: the US Food and Drug Administration (FDA) has been regularly criticized for being disproportionately strict and for requesting too much data. Clinical trials have thus become increasingly complex and time-consuming in the USA, leading to what has been termed "drug lag": prior to the Kefauver-Harris Amendments, an average of 40 new drugs were introduced each year, whereas the number dropped to 16 thereafter.

$\mathrm{T}$ he FDA is in a difficult position: being too strict ensures safety, but it slows down the approval process and can delay new drugs from benefiting patients. If this affects drugs for life-threatening diseases, delays can cost lives if there are no other treatment options. By way of example, during the early 1990s, activists in the US lobbied the FDA to ease its rules and expedite approval for the first generation of antiHIV drugs because so many AIDS/HIV patients were dying each year. On the other hand, not being strict enough can lead to public health problems if unsafe drugs enter the market. Prominent examples are Rezulin (troglitazone) and Vioxx (rofecoxib), both of which turned out to have unacceptable side effects.

\section{"Research began to leave the USA, first to Scandinavia and the UK, then to Eastern Europe and Latin America, and now to China and India"}

Yet, the FDA has an incentive to err on the side of caution: lives lost owing to drug delays usually results in fewer negative headlines than lives lost to dangerous drugs approved too soon. In addition, some of the FDA's regulations make clinical research more burdensome and costly even without improving human subjects protection or drug safety. It has been argued, for example, that requiring continued annual review by Institutional Review Boards (IRBs) even for minimal risk studies is simply not necessary.
In addition, multicentre studies are often reviewed by multiple local IRBs, a costly but redundant procedure. When clinical trials started to move abroad in the 1980s, stringent FDA regulations were a major factor, and still are today.

FDA regulation is not the only reason that so many clinical trials have moved to LMIC [1]. Because many new drugs confer only a small benefit over existing treatments, ever larger trials with increasing numbers of human subjects are required to measure improvements with statistical significance. This has become particularly relevant for so-called me-too drugs that are chemically very similar to those already on the market. Recruiting a sufficient number of patients therefore remains a major bottleneck for medical research, and companies have turned to LMIC to conduct trials because it is easier to find participants there. Moreover, operational costs in developing countries are low and there are large pools of "treatment-naive" patients, whereas in traditional research areas, the use of too much medication generates the risk of drugdrug interactions. Research began to leave the USA, first to Scandinavia and the UK, then to Eastern Europe and Latin America, and now to China and India (Fig 1). A recent investigation found that the 20 largest USbased pharmaceutical companies were conducting one-third of their clinical trials solely at foreign sites, while the majority of study sites are outside the USA [2].

W hen research by US companies is carried out in other countries, the FDA still has some responsibilities to ensure that these comply with ethical standards. But there are doubts whether all the US rules and regulations should be exported to other countries. "The US regulatory system is far from perfect, it has been criticized as being overworked and inefficient. Some would argue that it is not the best model to bring to lower income countries that don't have the resources we have”, said Sandra Alfano, Research Scientist at Yale School of Medicine and Chair of Yale's Institutional Review Board (IRB). Moreover, not all ethical principles have cross-cultural validity. Informed consent, for example, is a central concept for any research with human subjects. However, as Alfano remarked, "the principle of informed consent flows out of a Western philosophy of individual autonomy. That does not always translate well to other countries". An Eastern philosophy, she explained, may rather rely on community or family decision. "Individualism is not a universally embraced idea", she said. She therefore thinks that Western researchers "should be strongly encouraged to involve the local community in setting the agenda-deciding whether this is a good project to be done-and then helping to design the research protocol and addressing the question about who is the right body to give consent". This principle is referred to as "community-based participatory research", or CBPR. "CBPR is really the focus on everyone's mind, but it is very difficult to achieve”, Alfano said.

cc

"... the primary goal of conducting clinical trials in developing countries should be to address the health needs of the host population"

Despite cultural differences and the need to collaborate with local communities, there are guidelines for conducting research in international settings, notably the Declaration of Helsinki (DoH) by the World Medical Association (WMA), and the international guidelines for biomedical research involving human subjects by the International Organisation for Medical Sciences (CIOMS). They are not legally binding, but set out important standards nonetheless. "Many countries refer to the $\mathrm{DoH}$ in their legislation. But what is more important is that it is a document written by physicians and for physicians. There is a moral obligation for physicians to stick to these rules", said Ramin Parsa-Parsi, Head of the Department for International Affairs of the German Medical Association.

The DoH was adopted in 1964 and has since undergone seven revisions, most recently in October 2013. A major revision was made in 2000 in response to studies performed with US federal funds of motherchild transmission of HIV in developing countries, which denied effective medication to participants in the placebo control arm. The DoH, in its 2000 revision, accordingly greatly limited the use of placebos. However, some argued that this would restrict research too much and that long-term benefits would justify the use of placebos [3]. The FDA, 


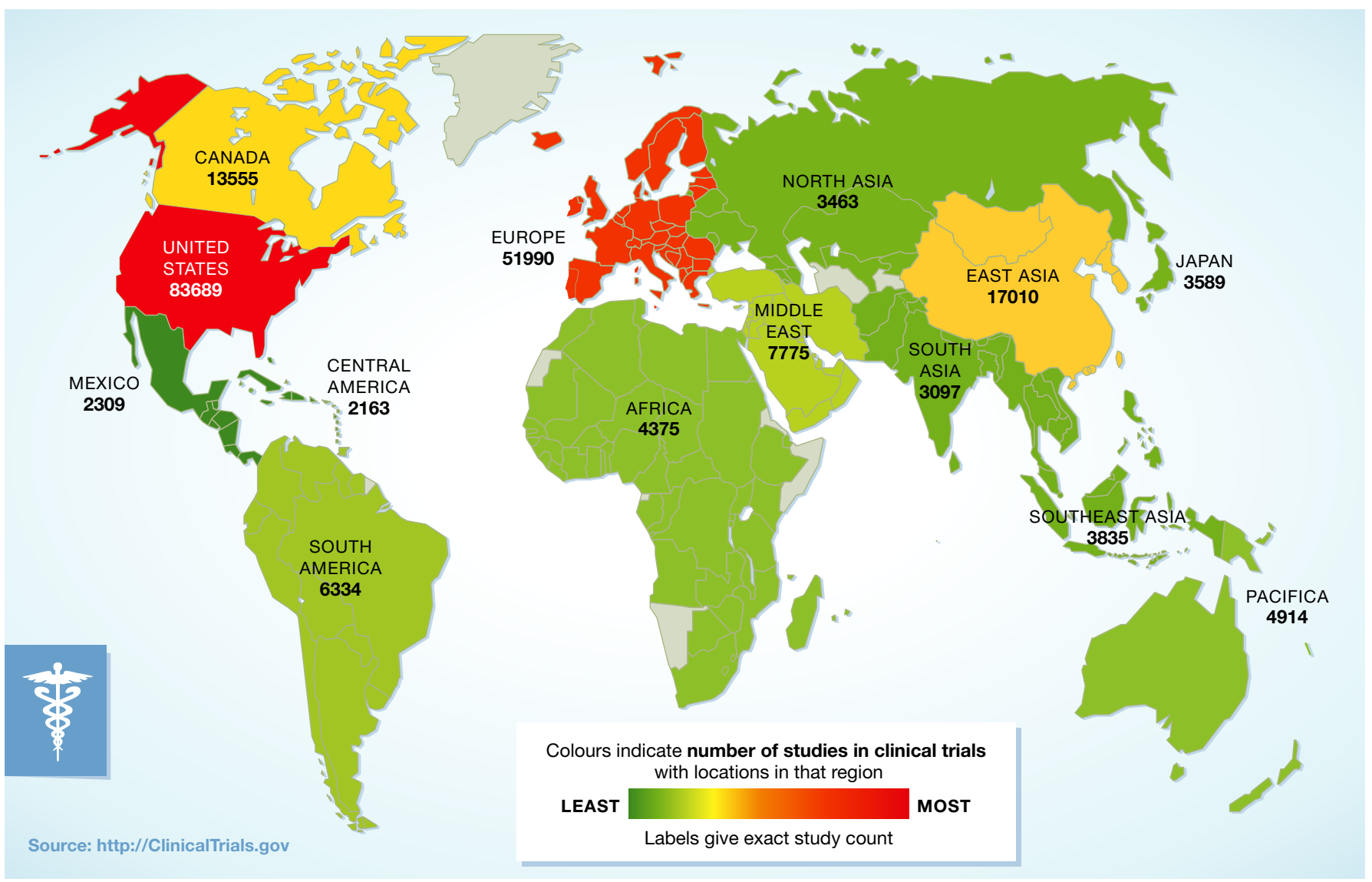

Figure 1. Distribution of study locations of all trials registered with ClinicalTrials.gov.

when regulating studies outside the USA, kept referring to older versions of the DoH and, in 2008, abandoned referring to it altogether at the expense of the "Harmonisation of Technical Requirements for Registration of Pharmaceuticals for Human Use Good Clinical Practice" (ICH-GCP) guidelines. "The ICH-GCP has been created by European, Japanese and US regulators in collaboration with the pharmaceutical industry. There are large differences in ethical standards between the $\mathrm{DoH}$ and ICH-GCP, particularly in the areas of placebo use and post-trial access”, ParsaParsi explained.

The newest revision of the $\mathrm{DoH}$, issued in late 2013, leaves the placebo paragraph essentially unchanged. The ICH-GCP regulation, on the other hand, is much more permissive: it explicitly states that the standard of care required to be provided to the control group depends on the population, meaning that control group subjects are only entitled to the standard of care they would otherwise receive locally. This is morally ambiguous. According to London, "you can't use the mere fact that people don't have access to an intervention that works to say that you don't have an obligation to provide them with something that works".

T he ongoing debate shows just how difficult it is to define ethical standards for research in international settings, to walk the narrow line between protecting research subjects and enabling research that contributes to the social good. According to London, the question of placebo-controlled trials should be viewed in the context of the research question studied. In his view, the primary goal of conducting clinical trials in developing countries should be to address the health needs of the host population. "In general, we should provide a high standard of care", he said, but maintained that there could be rare exceptions. Such exceptions might be in cases where placebo-controlled trials are particularly important to developing countries: for example, when testing a new intervention that is similar to but cheaper than existing drugs, or one that has some other characteristic that conveys particular advantages in LMIC. One such feature would be heat resistance, which would make it easier to transport medication to remote locations in tropical areas. In such cases, it would be reasonable to use a placebo control in the trial, as it will be difficult to compare the efficiency of the new product to the existing one.

A crucial aspect in the DoH and CIOMS guidelines concerns research on vulnerable groups. If people in developing countries belong to severely socio-economically disadvantaged populations, this would impact on their freedom to consent, making them susceptible to undue influence and therefore vulnerable. According to the $\mathrm{DoH}$, research in vulnerable groups is only justified, "if the research is responsive to the health needs or priorities of this group and the research cannot be carried out in a 
non-vulnerable group. In addition, this group should stand to benefit from the knowledge, practices or interventions that result from the research".

\section{"... taking advantage of people's deprivation to impose on them the risk of health research can be exploitive if it is not carefully planned and regulated"}

"We have discussed including, in addition to the current requirements which reflect the 'reasonable availability' approach, a wording on fair benefits", Parsa-Parsi said. Indeed, "fair benefits" is a hotly debated topic. Whereas the DoH and CIOMS base ethical acceptability mainly on responsiveness to health needs of the host community, others maintain that research is justified as long as the host community receives "fair benefits" in a more broadly defined sense. Such benefits would not have to be directly related to the research projects and might include employment and training for community members or the building of infrastructure [4]. However, after long debates and consultations with developing countries, a fair benefits option was not included in the current version of the DoH. "The possibility of exploitationthat you buy consent with money or other benefits - was considered too high by the WMA", Parsa-Parsi explained. London takes a similar view: "Ancillary benefits are important, but I don't think they can make up for lack of relevance of the science to local health needs", he said. For him, addressing the local health needs is the key issue. But ethical commitment should not stop there. "Just because I know the answer doesn't make you better off. That information then has to be translated into something from which you benefit concretely", he said. "You start by studying the health needs, you build up an information base needed to discover interventions that work and then you close the cycle of translation by making those interventions and that knowledge available to their communities."

Availability of the drugs developed remains another major issue. Medication is often far too costly people in LMIC to afford, but with time, that too can change. "People were worried that HIV studies carried out in LMIC were not relevant to local health needs because the drugs were too expensive”, London recalled. "Ten years later, many more people have access than what was originally expected." According to the WHO, the number of patients taking antiretroviral therapy has increased rapidly in LMIC since 2003 from just 400,000 to 11.7 million by the end of 2013 (http://www.who.int/gho/ hiv/epidemic_response/ART_text/en/).

$\mathrm{n}$ the meantime, other diseases are taking a terrible toll in LMIC, and it is not clear when new drugs will be cheap enough for wide use by their citizens. Hepatitis $C$, an infectious disease that can lead to liver cirrhosis, is on the rise globally, and developing countries carry the highest burden with infection rates as high as $11 \%$ in Egypt, $4.8 \%$ in Pakistan and $3.2 \%$ in China. During the past decade, research has led to the development of new drugs that work more effectively, in less time and with fewer side effects. Moreover, in contrast to the previous treatment regimens, these all-oral medications do not require regular interferon injections, making them ideal for use in remote settings. The drugs are just starting to come to market, with more in the pipeline, but their cost at the outset puts them out of reach for most people in developing countries. It is likely that, as in the case of AIDS medication, it will take long until they become available to most LMIC.

In many cases, therefore, studying local health needs will not necessarily generate immediate benefits for poorer communities. Nonetheless, studies that are responsive to local health needs will generate information that is necessary to improve public health in the long run. "When you ensure that the information is relevant to the local population, you build the foundation of knowledge necessary to generate beneficial interventions and policies, and this is a kind of benefit itself”, London commented. Making important new treatments available in LMIC should be considered a health priority, but this might take years or even decades at the current rate of progress. For participants in clinical trials, it will therefore be important to help them to bridge the gap between the end of the trial and the time the drug becomes available in their country.

$\mathrm{B}$ oth the CIOMS and the DoH require studies on vulnerable groups to address their health needs. But does research in LMIC address local health needs in reality? According to the 2014 report by the Access to Medicine Foundation, an international not-for-profit organization that ranks the top 20 research-based pharmaceutical companies with respect to their efforts to improve global access to medicine (http://www.accesstomedicineindex.org/), there is some promising development, but there is still a long way to go. "There is evidence of sustained commitment to R\&D for relevant diseases, with most companies having an R\&D strategy in place that explicitly takes patients in developing countries into account", the report says. More precisely, the index investigated research activities on 47 diseases that were regarded as most relevant to LMIC according to the WHO. Although a number of studies tackle these diseases, the research activities can mainly be attributed to only five of the 20 companies, and they concentrate on only five of the 47 diseases: diabetes, lower respiratory infections, hepatitis, HIV/AIDS and malaria. Neglected tropical diseases, as the report shows, remain neglected. In addition, the report criticises companies' lack of effort to make their products available to people in LMIC: "On average, products are registered in only [...] $8 \%$ of low-income countries covered by the Index."

Conducting clinical trials in LMIC is not intrinsically immoral. Indeed, trials sometimes represent the only medical treatment that patients in deprived settings can hope to receive, and can also provide other advantages at the personal, community and national levels. Nonetheless, taking advantage of people's deprivation to impose on them the risk of health research can be exploitive if it is not carefully planned and regulated. At the moment, there is such a variety of legislation that the conduct of trials differs widely, often depending on why a company decided to use a developing country in the first place. Trials that address locally relevant diseases and that will deliver affordable drugs and care for the local population are certainly to be lauded. Ones that do not provide immediate or obvious benefits locally are less laudable and must be assessed on a case-by-case basis.

\section{References}

1. Petryna A (2009) When Experiments Travel: Clinical Trials and the Global Search for Human Subjects. Princeton, NJ: Princeton University Press 
2. Glickman SW, McHutchison JG, Peterson ED, Cairns CB, Harrington RA, Califf RM, Schulman KA (2009) Ethical and scientific implications of the globalization of clinical research. $N$ Engl J Med 360: 816-823
3. Wolinsky H (2006) The battle of Helsinki: two troublesome paragraphs in the Declaration of Helsinki are causing a furore over medical research ethics. EMBO Rep 7: $670-672$
4. Schulz-Baldes A1, Vayena E, Biller-Andorno N (2007) Sharing benefits in international health research. Research-capacity building as an example of an indirect collective benefit. EMBO Rep 8: 8-13 